(13) Egeria.

\begin{tabular}{c|c|c}
\hline I 88I & $\Delta \alpha$ & $\Delta \delta$ \\
\hline März 23 & $+2^{5.64}$ & -30.0
\end{tabular}

(I 4) Irene.

Aug. I9 $|+3.17|+19.3$

$20+3.34+23.9$

$22+3.16+25.8$

$30+3.29+22.2$

(19) Fortuna.

Febr. $22|+14.48|-86.9$ $23+14.32-90.2$ $24 \mid+15.21-91.6$

(20) Massalia.

Juni $23|+2.05|+6.1$
(24) Themis.

\begin{tabular}{c|c|c}
\hline I $88 \mathrm{I}$ & $\Delta \alpha$ & $\Delta \delta$ \\
\hline März 23 & -0.50 & $+4 ! \cdot 2$
\end{tabular}

(32) Pomona.

Febr. 19 $1-0.2 \mid-\quad-1.5$ \begin{tabular}{l|l}
20 & -0.39 \\
21 & $-0.5 \mathrm{r}$
\end{tabular}$-0.4$

$22+0.18-\quad 1.5$

$23-0.06+0.6$

$24-0.25+2.2$

(37) Fides.

Nov. 20 $|-8.6 \mathrm{r}|-57.7$

(43) Ariadne.

Nov. $19|+2.45|+0.9$
(49) Pales.

\begin{tabular}{r|c|c}
\hline \multicolumn{1}{c|}{$88 \mathrm{1}$} & $\Delta \alpha$ & $\Delta \delta$ \\
\hline Febr.23 & -0.6 & $-50^{\prime \prime}$ \\
25 & $+\mathrm{I} .61$ & -33.5
\end{tabular}

(76) Freia.

Nov. I $6|-4.77|-28.3$ $19-5.25-25.9$ (92) Undina.

Jan. $2|+0.43|+x .1$ (I30) Electra.

Aug. $2|+1.56|+12.4$

(r34) Sophrosyne.

Aug. $25|-0.27|-7.1$

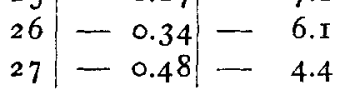

(x73) Ino.

\begin{tabular}{|c|c|c|c|c|}
\hline \multicolumn{2}{|c|}{$\mathrm{I} 88 \mathrm{I}$} & $\Delta \alpha$ & \multicolumn{2}{|c|}{$\Delta \delta$} \\
\hline Mai & 20 & +0.60 & - & 3.8 \\
\hline & 2 I & $+0.4 \mathrm{I}$ & - & 8.3 \\
\hline & 26 & +0.45 & - & 21.2 \\
\hline Juni & I & +0.95 & - & II.3 \\
\hline & I 8 & +0.67 & 一 & 22.5 \\
\hline & 21 & +0.69 & - & 20.6 \\
\hline & 22 & +0.30 & 一 & 23.4 \\
\hline Juli & 19 & +0.09 & - & 0.8 \\
\hline
\end{tabular}

Planetenbeobachtungen am Starke'schen Meridiankreise der Sternwarte OGyalla.

Mitgetheilt von Dr. Nicolaus von Konkoly.

Venus.

\begin{tabular}{|c|c|c|c|c|c|c|c|c|c|c|}
\hline I $88 \mathrm{I}$ & M. Z. OG. & $\alpha$ app. & Fäden & Rand & $\delta$ app. & Rand & Parall. & $\begin{array}{c}\mathrm{B}-\mathrm{R} \\
A \alpha\end{array}$ & $\begin{array}{c}\mathrm{B}-\mathrm{R} \\
\not \delta\end{array}$ & Bemerkungen \\
\hline Aug. 26 & $21^{h} 18^{m} 13^{s}$ & $7^{\mathrm{h}} 39^{\mathrm{m}} 50.99$ & I 5 & II & $+20^{\circ} 2 I^{\prime} \quad 4^{\prime \prime} \cdot 9$ & $\mathbf{N}$ & $3: 86$ & -0.32 & $+1 " 4$ & \\
\hline 27 & 21 I9 10 & - & - & - & +201257.1 & > & > & - & +2.2 & \\
\hline 29 & $\begin{array}{lll}21 & 20 & 47\end{array}$ & $754 \quad 23.46$ & 14 & II & +I95540.3 & > & 3.82 & +0.01 & +5.3 & \\
\hline Sept. I 8 & $\begin{array}{lll}21 & 36 & 59\end{array}$ & $930 \quad 29.85$ & I 4 & II & +I5 4 35.7 & , & 3.95 & -0.25 & +1.8 & \\
\hline
\end{tabular}

M a rs.

Aug. $26 \mid$\begin{tabular}{lll|l|l|l}
18 & 31 & I 6 & 4 & 53 & 36.54
\end{tabular}

\begin{tabular}{l|lll|lll}
27 & 18 & 29 & 46 & 4 & 56 & 3.04
\end{tabular}

\begin{tabular}{l|lll|lll}
30 & 18 & 28 & 40 & 5 & 3 & 43.45
\end{tabular}

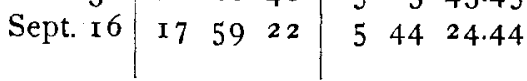

\begin{tabular}{|c|c|c|c|c|c|c|c|c|c|c|}
\hline$\times 5$ & I.M & $+2 I$ & $54 \quad 37.2$ & $\mathrm{~S}$ & 3.15 & - & 0.08 & - & 6.3 & \\
\hline 2 & M & $+2 I$ & 5940.6 & 3 & , & - & 0.50 & - & 5.2 & AR unsicher. \\
\hline$\times 5$ & I.II & +22 & 1352.2 & $>$ & 3.18 & - & 0.02 & - & $4 \cdot 3$ & \\
\hline I & II & +23 & 951.8 & , & $3 \cdot 3^{6}$ & + & 0.02 & - & 6.0 & \\
\hline
\end{tabular}

Jup it er.

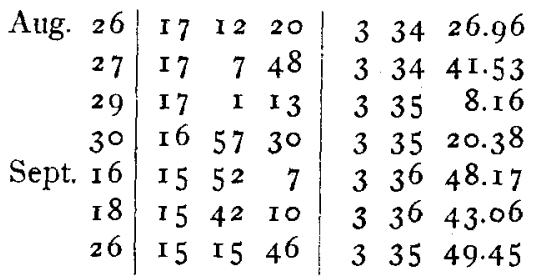

\begin{tabular}{|c|c|c|c|c|}
\hline$\times 5$ & I.II & +18 & 5 & 13.9 \\
\hline > & * & +18 & 5 & 54.5 \\
\hline " & , & +18 & 7 & 5.2 \\
\hline I 4 & > & +18 & 7 & 44.7 \\
\hline I 5 & $\gg$ & +18 & ro & $2 \times .0$ \\
\hline , & > & +18 & 9 & 43.0 \\
\hline ע & , & +18 & 5 & 39.6 \\
\hline
\end{tabular}

\begin{tabular}{|c|c|c|}
\hline 0.93 & +0.09 & $\cdots$ \\
\hline 》 & +0.02 & - \\
\hline 0.94 & +0.06 & \\
\hline 0.94 & +0.19 & t \\
\hline 0.99 & +0.20 & + \\
\hline » & +0.25 & + \\
\hline 1.0 & +0.23 & + \\
\hline
\end{tabular}

Saturn.

\begin{tabular}{rr|rrr|rrr} 
Aug. 26 & 16 & 21 & 5 & 2 & 43 & 5.27 \\
27 & 16 & 17 & 10 & 2 & 43 & 4.19 \\
29 & 16 & 5 & 19 & 2 & 43 & 1.29 \\
Sept. 16 & 14 & 56 & 47 & 2 & 41 & 19.71 \\
18 & 14 & 48 & 49 & 2 & 41 & 3.67 \\
28 & 14 & 15 & 15 & 2 & 39 & 5.68
\end{tabular}

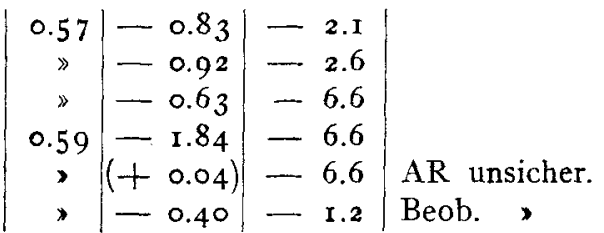

Dunst. 
$\mathrm{N}$ e p t u n.

\begin{tabular}{|c|c|c|c|c|c|c|c|c|c|c|}
\hline I $88 \mathrm{I}$ & M. Z. OG. & $\alpha$ app. & Fäden & Rand & $\delta$ app. & Rand & Parall. & $\begin{array}{c}\mathrm{B}-\mathrm{R} \\
\Delta \alpha\end{array}$ & $\begin{array}{c}\mathrm{B}-\mathrm{R} \\
\Delta \delta\end{array}$ & Bemerkungen \\
\hline Aug. 29 & $16^{\mathrm{h}} 25^{\mathrm{m}} 44^{\mathrm{s}}$ & $2^{\mathrm{h}} 5^{8 \mathrm{~m}_{2} 9^{5}} \cdot 15$ & I 4 & M & $+15^{\circ} 4^{\prime} 29^{\prime \prime} \cdot 9$ & $\mathbf{M}$ & O." 16 & +0.40 & $+5 * 3$ & \\
\hline Sept. I 6 & I5 I3 II & 25745.85 & 10 & * & $+15 \circ 24.4$ & > & » & +0.16 & +4.2 & \\
\hline I 8 & $15 \quad 5 \quad 12$ & $257 \quad 38.82$ & I 4 & , & +145950.3 & $>$ & » & -0.22 & +5.2 & \\
\hline
\end{tabular}

Sämmtliche Beobachtungen - mit Ausnahme der ち-Beobachtung vom 28. September, deren Fadenantritte registrirt wurden — sind mit »Auge-Ohr « erhalten, bei den Declinationen immer alle 4 Mikroskope abgelesen worden. Die mitgetheilten Declinationen sind schon mit dem angesetzten Betrage der Parallaxe - wobei $\pi=8.90$ angenommen wurde - corrigirt. Die Vergleichungen beziehen sich alle auf die Ephemeriden des Berliner Jahrbuches. In der 5. und 8. Columne ist angezeigt, von welchem Rande die Antritte beobachtet wurden, resp. welcher Rand am Mittelfaden eingestellt wurde.

Ich benutze die Gelegenheit zur gleichzeitigen Mittheilung einer, aus im August und September beobachteten
Culminationen von $\alpha$ Urs. min. abgeleiteten neuen Polhöhe der OGyallaer Sternwarte. Obzwar sich eben bei dieser Gelegenheit einige Mängel des Instrumentes und seiner Aufstellung zeigten, in Folge deren auch die einzelnen Werthe mehr als erwartet von einander abwichen, glaube ich doch, dass der nun gefundene Werth $\varphi=47^{\circ} 5^{\prime} 27^{\prime \prime} 3 \pm 0.4$ der Wahrheit bedeutend näher, als der bisher angenommene $(43.4)$, der sich ausserdem noch auf das frühere Observatorium bezog, kommt und keiner grossen Correction mehr bedarf. Es wird also bis $z \mathrm{u}$ einer nächsten Untersuchung vorderhand dieser Werth angenommen werden. OGyalla $188 \mathrm{r}$ Oct. 7 .

\section{Note on Meteoric Astronomy.}

In addition to the critical remarks of Dr. Rudolf Lehmann-Filhés on the history of meteoric astronomy (A. N. No. 2410), it will be interesting to refer to two memoirs in the Transactions of the American Philosophical Society Vol. VIII pp. 83-140, Philadelphia 1843 .

'The first memoir is by Prof. Benjamin Peirce, and is a criticism of Erman's theory. Professor Peirce points out Erman's onission, to which Dr. Lehmann-Filhés calls attention.

The second memoir is by Mr. Sears C. Walker. In this memoir there is given a full account of the observations, and the proposed theories of meteors; and formulae are also given for computing the orbits of these bodies. Walker's work will be found very complete and interesting.

U. S. Naval Observatory, Washington I 882 Febr. I 4 .

A. Hall.

\section{A nze ige.}

Die Herren Abonnenten, welche die Astronomischen Nachrichten ferner zu erhalten wïnschen, werden ersucht, ihre Bestellung und Vorausbezahlung auf den folgenden Band baldmöglichst einzusenden, wofern es der Expedition nicht bekannt ist, dass sie als ständige Abonnenten angesehen werden wollen.

Man pränumerirt bei der Expedition der Astronomischen Nachrichten, Sternwarte Kiel, mit nettö I 2 Mark für den Band von 24 Nummern nebst Inhaltsverzeichniss und Register. Für die von der Expedition nummerweise franco versandten Exemplare beträgt der Preis I 5 Mark. Einzelne Nummern werden zur Completirung, wenn sie vorräthig sind, zum Preise von 6o Pfennigen abgelassen.

Den Hauptdebit dieses Blattes hat, wie bisher, die Buchhandlung von W. Mauke Söhne in Hamburg.

Geschlossene Bände, von Band 100 an, können jederzeit von der Expedition, Sternwarte Kiel, zum Preise von I 2 Mark pro Band bezogen werden. Von den älteren Bänden sind noch Band 32-99 vorräthig und zu gleichem Preise bei Dr. C. F. W. Peters, Kiel, verkäuflich.

\footnotetext{
Berichtigurgen zu Nr. 2387 pag. 173 Z. 14 v. o.: Das Datum statt June 18 l. June Io.

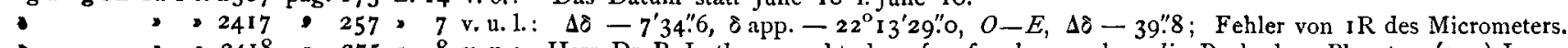

- $\quad 2418,275,8$ v. u.: Herr Dr. R. Luther macht darauf aufmerksam, dass die Beob. dem Planeten (I39) Juewa zugehören, die pag. 277 fortgesetzt werden.
}

\section{In h a l t :}

Zu Nr. 2422. H. Kobold. Beobachtungen kleiner Planeten am Astrophysikalischen Observatorium zu OGyalla. 337. - N. v. Konkoly. Planeten. beobachtungen am Starke'schen Meridiankreise der Sternwarte OGyalla. 349. - A. Hall. Note on Meteoric Astronomy. $351 .-$ Anzeige. $35 \mathrm{r},-$ Berichtigungen. $35 \mathrm{I}$. 